\title{
Quantum Jump Approach to Stimulated Absorption and Emission
}

\author{
Chang Jae Lee \\ Deparment of Chemistry, Stmmoon University, Asan 336-708, Korea. E-mail: coolcjl@summoon.ac.kr \\ Received Febriary 5, 2006
}

\begin{abstract}
In this paper a new theory is presented to treat the problem of stimulated absorption and emission of photons between energy levels from the standpoint of discrete quantum jumps. In order to implement the theory a scheme to avoid the quantum Zeno effect is proposed. Numerical simulations are performed to demonstrate that this approach does not contradict the principles of the standard wave mechanics. It is shown that with this approach one can obtain photon observation statistics as well.
\end{abstract}

Key Words : Quantum jump, Monte carlo wave function. Wave function collapse, Stimulated absorption, Stimulated emission

\section{Introduction}

The canonical interpretation of quantum mechanics provides us with an expectation value of an observable, which is equal to an ensemble average over similarly prepared systems. ${ }^{1}$ However, advances in experimental techniques have allowed the close examination of a single atom or ion. ${ }^{2}$ The single atom experiments we are interested in are those which dramatically reveal Bohr's quantum jumps between energy levels ${ }^{3}$ that would have been smeared out by the ensemble averaging. These features (also known as the collapse of the wave function) may not be accounted for by the Schrödinger equation that can only predict continuous evolution of the wave function.

Beginning early 1990 s several research groups developed theories to treat quantum jumps using stochastic methods. ${ }^{+}$ These are best known as the Monte-Carlo Wave Function (MCWF) theories, and recently they have been applied to quantum computing ${ }^{5}$ and atom lithography. ${ }^{6}$ The original motivation of the MCWF theories is concerned with the dissipative process of spontaneous emission. Atoms spontaneously emit photons at random directions and times, so theses theories could naturally be based on stochastic techniques. When the atom is subject to a coherent driving field like laser, coherent processes of stimulated absorption/ emission as well as incoherent spontaneous emission are present. In the MCWF method the atom evolves coherently and continuously according to the time-dependent Schrödinger equation until it spontaneously emits a photon and the atomic state makes a discrete jump, which is treated stochastically. Since quantum jumps may also occur in stimulated absorption and emission, however, the MCWF methods may not consistently treat the processes.

The purpose of this paper is to provide a means to remedy the defect of the MCWF theories. Since the problem of spontaneous emission has already been addressed, we will concentrate on the stimulated processes. In fact, there are several methods, including cavity-QED experiments, ${ }^{7}$ for suppressing spontaneous emission. Thus, considering only the stimulated processes is not unrealistic and has merits on its own. We will attempt to incorporate in the Schrödinger equation the quantum jumps due to these processes.

\section{Simulation Method}

To capture the essential point of the matter we consider the simplest case of shining a resonant single mode light to a two-level atom. ${ }^{8}$ We first sketch how the problem is handled traditionally. Let the lower and upper states of the atom be denoted as $|1\rangle$ and $|2\rangle$. The evolution of the atomic state is governed by the Schrödinger equation

$$
\frac{d}{d t}|\psi(t)\rangle=-\frac{i}{\hbar} H|\psi(t)\rangle
$$

with the Hamiltonian

$$
H=\frac{\hbar \omega_{-}}{2} \sigma_{z}+\frac{\hbar \Omega}{2}\left(\sigma^{-i \omega_{i} t} \sigma_{-}+\sigma^{i \omega_{A} t} \sigma_{-}\right),
$$

where $\omega_{A}$ is the resonance frequency between the two atomic energy levels, $\Omega$ is the Rabi frequency, $\sigma_{1}=|2\rangle\langle 1|$, $\sigma_{-}=|1\rangle\langle 2|$, and $\sigma_{z}=|2\rangle\langle 2|-| 1\rangle\langle 1|$. The solution of Eq. (1) is well known. ${ }^{9}$ If initially the atom is in the lower state, $\psi(0)\rangle=|1\rangle$, the interaction of the atom with the light puts the atom in a coherent superposition state $|\psi(t)\rangle=c_{1}(t)|1\rangle$ $+c_{2}(t)|2\rangle$. When it is substituted in Eq. (1) with the rotatingwave approximation, we have the probabilities of finding the atom in the respective states

$$
\begin{aligned}
& P_{1}(t)=\left|c_{1}(t)\right|^{2}=\cos ^{2} \frac{\Omega t}{2}, \\
& P_{2}(t)=\left|c_{2}(t)\right|^{2}=\sin ^{2} \frac{\Omega t}{2} .
\end{aligned}
$$

Note that the state of the atom evolves continuously between the lower and the upper states, giving the celebrated Rabi oscillation. It does not show when the atom absorbs or emits a photon, however.

In order to simulate discrete quantum jumps we divide the total observation time $T_{\text {obs }}$ into small segments, each having $\Delta t$. For each time segment we propagate the atom according to the Schrödinger equation, and at the end of the segment 
we observe if a photon is absorbed or emitted. Such an observation makes the wave function collapse. But what is the collapse probability? We make the ansal that $P_{j}(t)$, $(j=1,2)$ in $\mathrm{Fq} .(3)$ is the probability for the wave function collapse $|\psi(t)\rangle \rightarrow|j\rangle$. We further assume that the collapse occurs instantancously. ${ }^{10}$ Since $P_{1}(t)=1-P_{2}(t)$ at any lime $t$, we need to consider only the probability $P_{2}(t)$. Our simulation takes place at discrete times $t_{n}=n \Delta t,(n=1,2$, $3, \ldots)$. For each $t_{1}$ we generate a random number $r_{n}$ and compare it with $P_{2}(t)$. We take that $P_{2}\left(t_{n}\right) \geq r_{n}$ as the condition for the collapse $\left|\psi\left(t_{n}\right)\right\rangle \rightarrow|2\rangle$ to cecur. Oherwise, we have the collapse $\left|\psi /\left(t_{t}\right)\right\rangle \rightarrow|1\rangle$, instead. Then we have four possible cases of quantum jump at $t=t \mathrm{~s}$ and photon absorption/emission during the interval $\left(t_{n-1}, t_{n}\right)$ :

(1) $P_{2}\left(t_{n-1}\right)<r_{n-1}$ and $P_{2}\left(t_{n i}\right)<r_{n}:|\psi\rangle \rightarrow|1\rangle$, no absorption.

(2) $P_{2}\left(t_{n-1}\right)<r_{n-1}$ and $P_{2}\left(t_{n t}\right) \geq r_{n t}:|\psi\rangle \rightarrow|2\rangle$, absorption.

(3) $P_{2}\left(t_{n-1}\right) \geq r_{n-1}$ and $P_{2}\left(t_{n}\right) \geq r_{n} ;|\psi\rangle \rightarrow|2\rangle$, no emission.

(4) $P_{2}\left(t_{n-1}\right) \geq r_{n-1}$ and $P_{2}\left(t_{n}\right)<r_{n} ;|\psi\rangle \rightarrow|1\rangle$, emission.

These four regions are schematically shown in Figure 1 . However, it should not be construed that the wave function exists only as either $|1\rangle$ or $|2\rangle$. As mentioned earlier, the

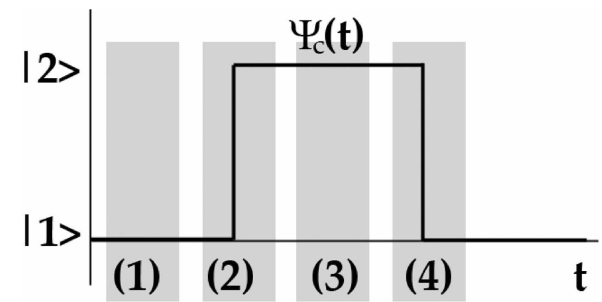

Figure 1. Four typical regions of measurement history. $\psi_{t}(t)$ denotes the collapsed wave function. In region (I) the atom is found to be in the lower state as in the previous measurement, so no photon is absorbed. The atom makes a transition to the upper state in region (2), and a photon is absorbed. The atom is still at the upper state in region (3), so no photon is emitted. In region (4) the atom returns to the lower state, emitting a photon.
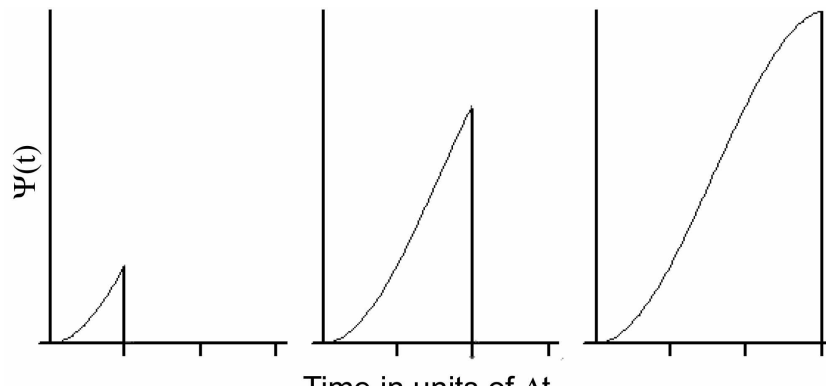

Time in units of $\Delta t$

Figure 2. The proposed measurement scheme to avoid the quantum /cho effect. Successive measurements are made after the atom evolves for integer multiples of $\Delta t$, each time starting from $t-0$. wave function is in coherent superposition states until a measurement is made, and we plot the collapsed wave finction as a result of measurements at discrete times.

There is one caveat with the measurement intervals. Since the wave function collapses each time a measurement is made, the atom never has a chance to leave the lower state if it is continuously observed (i.e. $\Delta t \rightarrow 0$ ). For finite $\Delta t$ the probability of having made a transition after $T_{\text {sto }}$ is reduced. This is known as the quantum 7eno effect ${ }^{11}$ and is experimentally verifiable. '2 Clearly, a scheme to avoid this effect is necessary to correctly observe (and simulate) light absorption/emission. One such a scheme is shown in Figure 2, where we make a series of measurements following the atomic evolution, each time with increasing duration starting from $t=0$ to $n \Delta t,(n=1,2,3, \ldots, N)$. It means that the atom needs to be prepared afresh for each measurement. Such a series of $N$ measurements conslitutes a guantum trajectory for the atom.

\section{Results and Discussion}

In our simulation the Rabi frequency $\Omega=5 \times 10^{\prime}$, the time

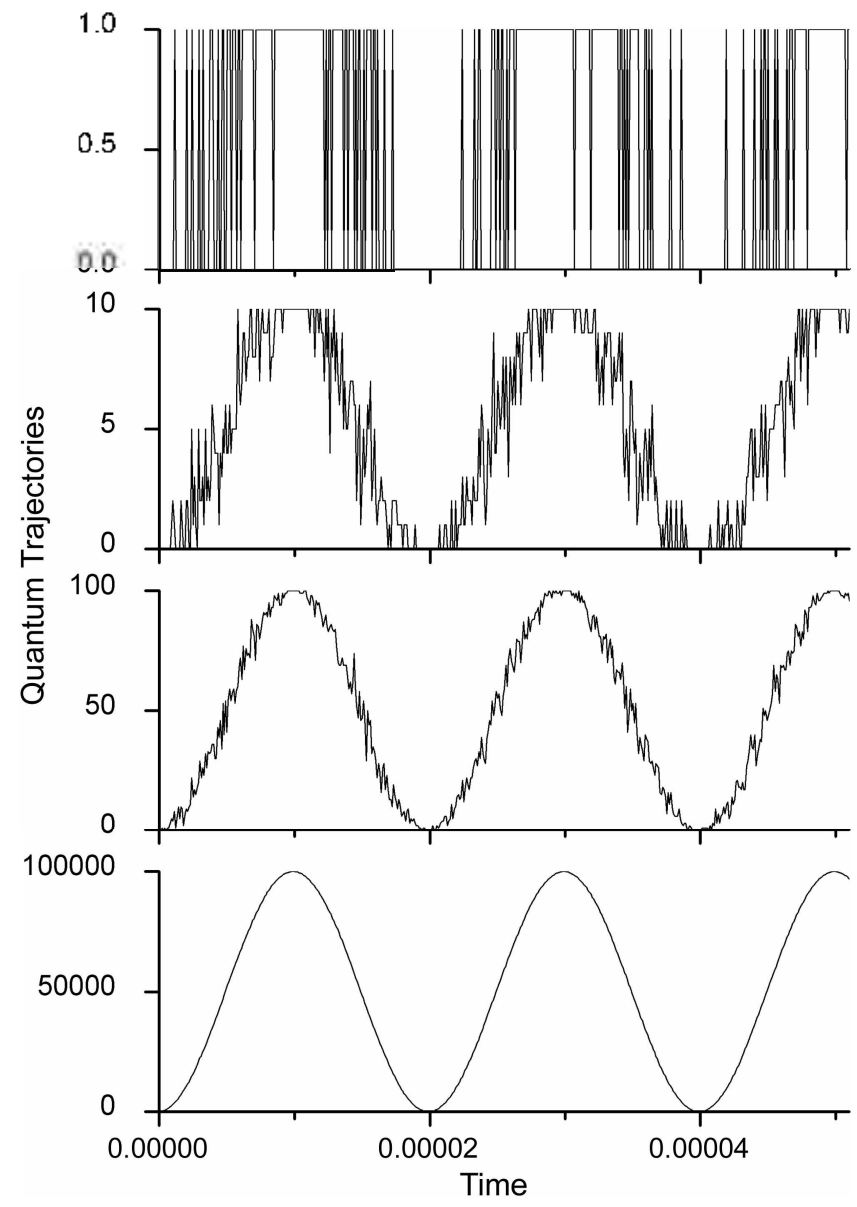

Figure 3. Simulated measurement histories with $1,10,100$, and $10^{8}$ atoms (from top to bottom). The one atom history shows discrete quantum jumps, which are gradually smeared out and the oscillatory structure becomes more evident as the number of atoms increases. 


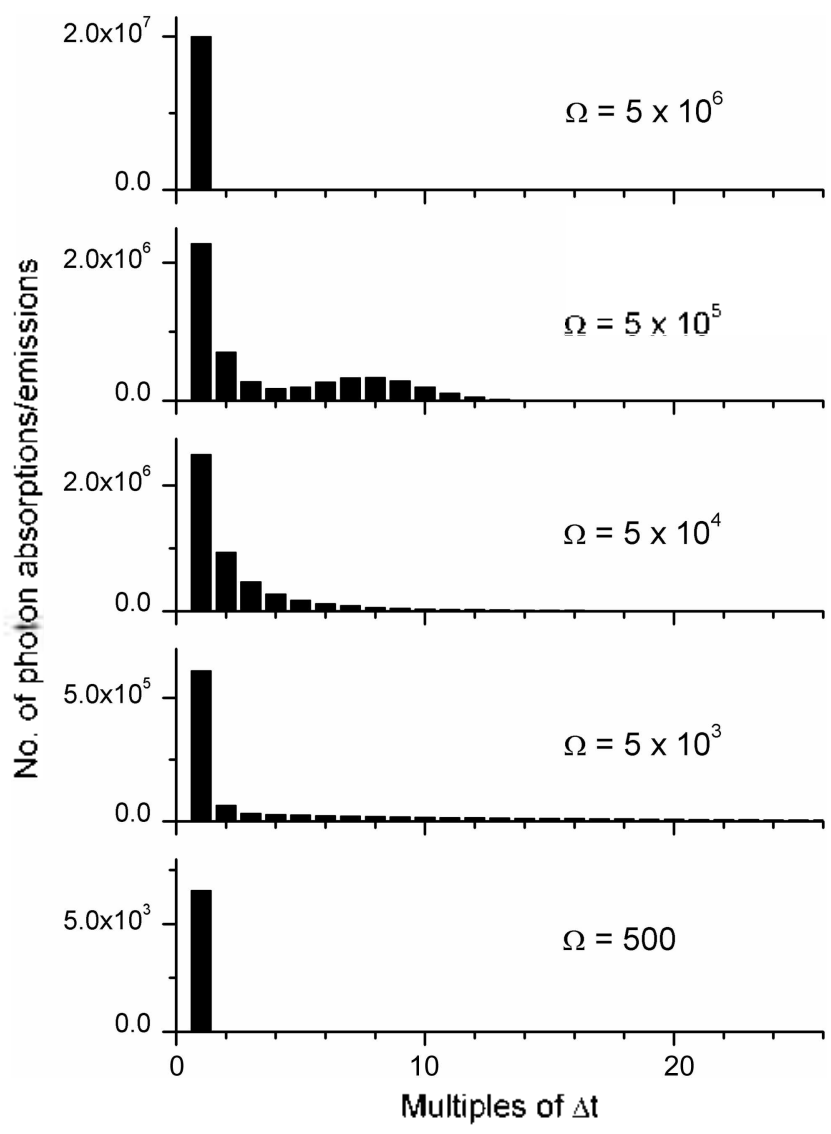

Figure 4. The wailing-time distribution as the Rabi frequency is varied. In all cases considered, the back-to-back absorption and emission is the most pronounced. Except for two extreme cases there is one absorption or emission per approximately $4 \Delta t$.

increment $\Delta t=1.0 \times 10^{7}$ (both the Rabi frecuency and time are in arbitrary units such that frequency $\times$ time $=1$ ), and the number of time segments $N=500$. Thus, the overall observation time $T_{\text {olls }}=N \Delta t=5 \times 10^{-5}$. Figure 3 shows the simulated history of observations of photon absorption/ emission as a function of time. The number of atoms from top to bottom in the simulation is $1,10,100$, and $10^{5}$. As the number of atoms increases, the oscillatory structure of the Rabi nutation becomes manifest. One more thing to note is that the one-atom quantum trajectory shows that the photon absorptions/emissions are concentrated in the region where $P_{1}(t) \approx P_{2}(t)$ despite the stochastic nature of the simulation. In a sense, the processes may be regarded as "bunched". ${ }^{13}$ Then it would be interesting to calculate the distribution of waiting times defined by the time intervals for the atom to absorb or emit a photon. Figure 4 shows the simulated waiting-time distribution with different $\Omega$ values kecping other parameters the same. Although the details vary, the distributions are sharply peaked about a $\mathrm{few}$ multiples of $\Delta t$. We have found that for $\Omega$ values not too high or too low there is approximately one absorption or emission per $4 \Delta t$.

\section{Conclusions}

In conclusion, we have shown that the quantum jump approach can be applied to the problem of stimulated absorption/emission without contradicting the principles of the standard wave mechanics. We have demonstrated this by recovering the Rabi oseillation, and the approach even allows us to obtain photon observation statistics, which is not possible with the usual quantum theory. Finally, we have proposed a scheme to avoid the perplexing quantum 7eno effect.

\section{References}

I. See, lor example: Ballentine, I. Г. Rex. Hod. Phus. 1970, 42, 358 See also his textbook: Quantum Mechanics; Prentice-Hall: Englewood Clills, 1990.

2. For recent reviews, see: (a) Rempe, G. Phnsics World 2000, December, 37. (b) Cirac, I. I.; Zoller, P. Phosics Today 2004, March, 38 .

3. (a) Dehmelt. II. Buff. Am. Phs. Soc. 1975, 20, 60. (b) Nagourney, W.: Sandberg, J: Dehmelt, It. Phys: Rev. Lett. 1986, 56, 2797. (c) Sauter, I; Neuhauser, W.: Blatt, R.; Joschek. I. E. Plns. Rev Lett. 1986, 57, 1696. (c) Bergquist, I. C.; Hulet. R. G.; Itano, W. M.; Wineland, D. J. Phis. Rev. Letr. 1986.57. 1699.

4. (a) Gisin, N, Percival, 1. C. J. Plws. 1992, A25. 5677. (h) Camichacl, H. J. An Open Systems Approwh to Quantum Optics: Springer: Berlin, 1993. (c) Dalibard, J. Castin, Y: Molmer, K. Phys. Rov Lett. 1992, 68, 580. (d) Gatdiner, C. W.; Parkins, A. S.; Zoller, P. Phws, Rov. 1992, A46, 4363. (e) Wisennan, H. M.; Milburn, G. J. Phis. Rev, 1993, A47, 1652. (f) Plenio, M. B.; Knight. P. I. Rev Mod. Plws. 1998. 70. 101.

5. Pary, E.; Calarco, T.; Zoller, P. IEEE Trans. Nonotechnot 2004, 3. 10. and reterences eiled therein.

6. (a) Lee, C. I. Phis. Rev 2000, A61, 063604, (b) Lee, C. I. Bull. Korean Chem. Sox 2003, 24,600.

7. For a recent review, see: Walther, H. Cavity $Q E D$ In Enculofodia of Mockern Optics; Guenther. R. D.; Steel, D. G: Bayvel, I., ए,ds.; Filsevier: Oxford, 2005: Vol. 4, p 218.

8. Allen, [..; Fberly, J. I]. Optical Resonance and Two-Level Atoms: Dover: New York, 1987.

9. Rabi, l. I. Plns. Rev: $1937,57,652$.

10. See, for example: Greenstein, G; Zajonc, A. G. The Qnantwm Challenge; , knes and Bartlett Publishers: Sudburry, 1997.

11. Misra, B.; Sudarshan, E. C. C. J. Wath Phws. 1977, 18, 756 .

12. Itano, W. M.: Heinzen, D. J.a Bollinger, J. J.; Weinland, D. J. Phys. Rev $1990, A 41,2295$

13. Hastbury-Brown, R.: Twiss, R. Q. Name 1956, $/ 77,27$, 